\title{
Study on the Personalized Learning Model of Learner-Learning Resource Matching
}

\author{
Lijuan Zhou, Feifei Zhang, Shudong Zhang, and Min Xu
}

\begin{abstract}
With the development of service integration technology, online learning platforms have gathered a large number of learning resources, causing learners to get lost in a variety of course information and it is difficult to obtain learning resources that match their own needs. The proposal of personalized learning gives the problem a direction to solve. However, current personalized learning resource recommendation services facing problems such as excessive candidate resources, sparse history and cold starts. In addition, the learning resources provided also show problems of "difficult or easy, uneven quality". For this article researches the personalized learning recommendation model of learner-learning resource matching. The main content includes three parts: First, build a demand model based on learner registration information, learning behavior and other data. Second, analyze the access behavior of learning resources and assess their quality. Third, calculate the matching degree between learners and learning resources based on the demand model and the quality information of the learning resources, and recommend them.
\end{abstract}

Index Terms-Collaborative filtering, demand models, learning resources, personalized learning.

\section{INTRODUCTION}

With the advancement of social science and technology, people have entered a new era of Internet. The development of this era requires a large number of personalized talents with innovative capabilities. Cultivation of the talent need to pay attention to personality characteristics of learners, realize the personalized learning. Personalized learning refers to the activities based on the learner's original knowledge and experience and individual characteristics, with internal needs as the core, and the goal of free, full and harmonious development of learners' capabilities and personalities [1]. Traditional education, dominated by classroom teaching system, provides different students with the same learning resources, while ignoring the individual learning needs of learners [2]. Online education is a teaching method centered on the online learning platform, which implement the student-oriented and teacher-led education concept, learners can find the resources they need through the platform to meet their individual needs.

To a certain extent, online education has realized the desire of many people to keep learning, meet the learning needs of

Manuscript received February 17, 2020; revised November 12, 2020 This research was supported by National Key R\&D Program of China (2019YFB1406301).

The authors are with the College of Information Engineering, Capital Normal University, Beijing, China (corresponding author: Shudong Zhang; e-mail: zhoulijuan@cnu.edu.cn, 2181002035@cnu.edu.cn, zhangshudong@cnu.edu.cn, xumin@cnu.edu.cn). learners and help them to increase their knowledge and capabilities. However, with the gradual expansion of online learning platforms and the continuous increase of learning channels, learning resources have started to accumulate, and the emergence of a large number of resources makes it difficult for people to find the learning resources they want from search results [3]. In order to enable learners to obtain learning resources that meet their own needs in a short time, personalized learning recommendation systems have emerged at the historic moment, such as MOOC [4], NetEase Cloud Classes and NetEase Open Classes, etc. It can be according to the basic personal information of learners when registering, long-term and short-term learning behavior and evaluation data, dig into the background of learning, learning style, learning needs and other personal characteristics, and recommend to their needs of learning resources, greatly reduce the learner's time to look for resources, improve the learning efficiency.

The structure of the rest of this article is as follows: Section II introduces the current personalized learning recommendation methods, analyzes their advantages and disadvantages, Section III details the learner-learning resource matching personalized learning model and Section IV is the conclusion of this article.

\section{RELATED WORK}

This section mainly introduces the working principle of the current popular personalized learning recommendation technology, analyzes their advantages and disadvantages and fully understands the research status of personalized recommendation technology.

With the development and progress of computer technology, at the beginning of the 21 st century, foreign researchers realized the importance of personalized recommendation in the field of network learning and began to study the personalized recommendation system in network learning [5]. Personalized learning recommendation technology is the embodiment of the latest education concepts. It focuses on the learner and recommends a series of learning resources to the learner to meet their current learning needs based on the learner's personal basic information and historical learning records. Currently, there are three main types of recommendation algorithms applied to personalized learning: Content-based recommendation algorithm, Collaborative filtering recommendation algorithm and Hybrid recommendation algorithm. In order to provide accurate and effective learning resources for learners, many researchers have studied the personalized learning mode in these methods and achieved ideal results. For example, Aher 
and Lobo recommended courses for freshmen with k-means and Apriori based on learners' historical data, and got better results [6]. Ghauth and Abdullah proposed a new content-based recommendation algorithm integrating excellent learner scores, which can recommend high-quality learning resources for learners [7]. Based on the computing perspective of context awareness, Khribi MK et al. proposed a context-aware recommendation system to promote learners to effectively conduct personalized online learning [8]. Salehi M et al. proposed a recommendation method based on sequential pattern mining and multidimensional attribute collaborative filtering, and adopted an improved PrefixSpan algorithm to discover potential patterns in resource access [9]. Saranya KG et al. proposed a collaborative filtering method based on rough sets to predict users' lost news category rating values, and a new detection method to improve the ranking of news items [10]. S.haskaran et al. mined the sequences obtained from AprioriAll algorithm, analyzed learners' behaviors and preferences, and realized customized recommendation of learning content [11]. Wang et al. labeled the curriculum structure and content based on the learner's characteristic model to realize the personalized recommendation function [12]. Scherch et al. used ontology technology to model learners and learning resources, and used semantic relationships to achieve personalized recommendation effect [13].

In summary, many researchers have made great progress in the research of personalized recommendation technology from different perspectives, mainly focusing on the establishment of models, adaptive mechanisms, recommendation strategies and other aspects, based on personalized learning model. However, there are few researches on the network platform, and personalized learning recommendation is facing many problems due to the rapid growth of learners and learning resource data.

\section{RESEARCH ON PERSONALIZED LEARNING RECOMMENDATION MODEL}

In the context of big data, the online learning platform gathers many learning resources with different or similar functions and attracts a large number of learners. With the increase of learning resources and learners, personalized learning recommendation systems is faced with too many candidate resources, sparse historical records, cold start and other problems. In the face of learning resources with the same or similar functions, how to choose high-quality resources has become the focus of research.

Therefore, this paper studies the personalized learning model of learner-learning resource matching. For new registered users, due to insufficient behavioral information on the platform, this model can analyze the personal information and interest preferences filled in by learners, find similar users by using collaborative filtering algorithm, and recommend learning resources to target learners according to similarity and the popularity of resources. For learners who have been using the learning platform for a long time, the platform stores their personalized behavioral data. Based on these data, a demand model can be established, and according to the matching degree between the demand model and the learning resources, the resources that meet the learning needs of learners can be recommended to learners. As shown in Fig. 1.

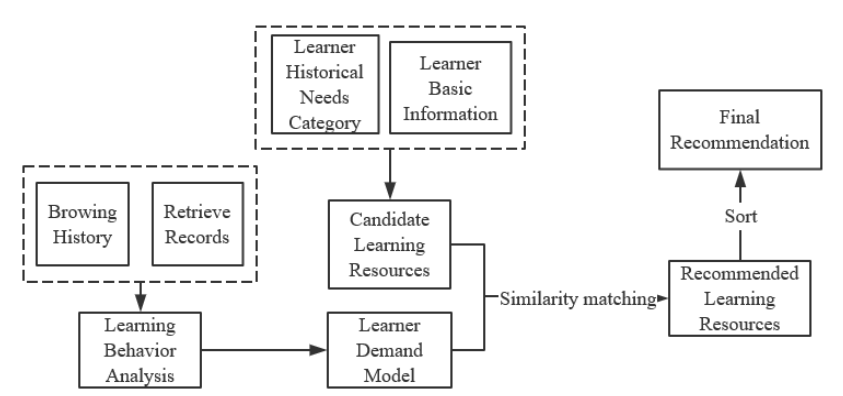

Fig. 1. Personalized resource recommendation.

\section{A. Learning Resource Description}

The learning resource expressions on the Internet are roughly divided into three types: text documents, audio and video. With the development of big data and Internet technologies, learning resources have shown rapid growth in number and scattered resources. In order to better manage and categorize learning resources and reduce the space for learner-learning resource matching, formalized descriptions of learning resources are needed.

The current methods for describing resources are mainly based on two types: content and classification [14]. Usually, the method by which domain experts add tags to the learning resources in the resource library is a content-based method, by the existing clustering algorithms (such as support vector machine (SVM), Naive Bayes or K-Means method) adaptive generation is not the same kind of method is based on the classification method. However, in the open network, the platform can continuously upload new learning resources, and these resources will enter the platform database after passing the review. The addition of these resources raises two issues:

1) The category of new resources. To ensure that these resources are accurately classified and used, we need to cluster the resources in the database each time a new resource is generated. With the increase of resources, each clustering takes a lot of time, so we need to cluster the existing resources in the library and determine the center point of the class. When a new resource appears, we only need to calculate the similarity between the new resource and the center point. The classification of new resources can be identified, greatly reducing computation time. When a new resource appears, we only need to calculate the similarity distance between the new resource and each central point to confirm the category of the new resource, which greatly reduces the calculation time.

2) The quality of resources in an open network environment. The emergence of a large number of learning resources with the same or similar content makes it difficult for learners to choose. By analyzing the behavior of resources being accessed and setting different weights, the quality of each learning resource can be evaluated objectively. For example, the quality of a video resource can be assessed by the number of views, favorites, downloads, and comments from some users. 
Definition 1: Use triples $\mathrm{R}$ to represent learning resources: $\mathrm{R}=$ (Item_type, Item_key, Item_hard). Among them, Item_type indicates the presentation type of the resource, such as document or audio or video; Item_key indicates resource keywords information and Item_hard indicates resource difficulty information. The main content of each dimension information is shown in Table I.

TABLE I: LEARNING RESOURCES REPRESENT INFORMATION

\begin{tabular}{lll}
\hline \hline Type of Data & Main Content & Get method \\
\hline Type information & $\begin{array}{l}\text { Document or audio or } \\
\text { video }\end{array}$ & Resource performance \\
Keywords & $\begin{array}{l}\text { Keywords information } \\
\text { set and corresponding }\end{array}$ & $\begin{array}{l}\text { Keyword extraction and } \\
\text { weight calculation }\end{array}$ \\
information & weights & Domain expert \\
Difficulty & Difficult, moderate, & assessment \\
information & easy & \\
\hline \hline
\end{tabular}

\section{B. Learner Need Description}

There are differences in the learner's learning ability, interest habits, learning foundation, subjective effort, etc. These individual differences will significantly affect learners learning behavior and learning quality, fully understand the learner's personal information, and establish a suitable user model for the learner only by carefully designing the learner profile can we effectively develop personalized learning services [14].

The learner's personalized information includes basic personal information and learning information. Personal basic information is the self-filled information of learners during registration, such as name, gender, occupation, interest preferences, etc.; learning information is historical learning behaviors stored on online learning platforms, such as resource browsing records and resource retrieval records, and they can reflect the learner's learning needs, learning style and learning ability.

Definition 2: Use a five-tuple B to represent the basic information of the learners: B = (Stu_name, Stu_sex, Stu_age, Stu_trad, Stu_prefer), which respectively represent the learner's name, gender, age, occupation, and interest preferences.

Definition 3: Use the triples $S$ to represent the learning information of the learner: $\mathrm{S}=$ (Stu_bias, Stu_key, Stu_hard). Among them, Stu_bias represents the type of resource performance of the learner's historical preferences. For example, in the learner's historical learning behavior, $80 \%$ of video resources are used, and we can get 〈Video: 0.8$\rangle$. Stu_key is key information of the learner's needs and Stu_hard represents learning ability information. As shown in Table II.

\begin{tabular}{lll}
\multicolumn{3}{c}{ TABLE II: LEARNER INFORMATION } \\
\hline \hline Type of Data & Main Content & Get method \\
\hline $\begin{array}{l}\text { Preferred resource } \\
\text { representation }\end{array}$ & Document and audio and video & $\begin{array}{l}\text { The historical } \\
\text { record }\end{array}$ \\
$\begin{array}{l}\text { Demand key } \\
\text { Information }\end{array}$ & Key words and weights of & $\begin{array}{l}\text { History keyword } \\
\text { extraction }\end{array}$ \\
$\begin{array}{l}\text { Learning ability } \\
\text { information }\end{array}$ & Strong, medium, weak & $\begin{array}{l}\text { Daily assessment } \\
\text { of learners }\end{array}$ \\
\hline \hline
\end{tabular}

E-learning platforms have the following characteristics: dynamic requirements. Learners' learning records appear at different times in succession. The platform can obtain the learning needs dynamically according to the learner's behavior and provide the corresponding learning resources according to the needs. To support this feature, we introduce a timestamp to represent the moment at which the learner's learning record is presented. Therefore, the time decay function can be used to calculate each weight of the learner's demand information. The closer the learning record is to the current time, the greater the need for this type of resource. The formula for calculating the time attenuation function is shown in (1):

$$
f\left(t_{1}-t_{2}\right)=\exp \left(-\alpha\left|t_{1}-t_{2}\right|\right)
$$

where $\alpha \geq 0$ is a non-negative decay constant, $t_{1}$ is the current moment, and $t_{2}$ is the moment when the learner needs to be raised.

However, with the passage of time, the learning platform becomes more and more record, early and low-weighted records need to be deleted based on the timestamps of each history record.

\section{Learner-Learning Resource Matching Personalized Learning Resource Recommendation}

At present, the more mature personalized recommendation method is the collaborative filtering method based on neighborhood. This method is mainly divided into two types, item-based collaborative filtering algorithm and user-based collaborative filtering algorithm [15]. The personalized learning mode of learner-learning resource matching directly matches the historical learning needs of users with the learning resources, and has a better solution to the problem of cold startup:

1) For learners to A new registration, through A personal basic information and the interest preference information, find other learners of high similarity, recorded as $\left\{S_{1}\right.$, $\left.s_{2}, \ldots, s_{\mathrm{k}}\right\}$. According to the historical learning behavior of these learners, the learning resources with high learning frequency are recommended to A.

2) For learners who often use the learning platform, we can first determine the candidate learning resources according to the historical learning records of learners, and then establish the learner demand model to match the demand model with the resources in the candidate learning resources.

Algorithm 1: Based on the foregoing definition of the learner's learning needs and the basic characteristics of the resources, the method of calculating the cosine angle of the vector space is used to measure the similarity between the needs of the learners and the learning resources. The calculation formula is shown in (2):

$$
\operatorname{sim}\left(s_{i}, r_{j}\right)=\cos \theta=\frac{s_{i} * r_{j}}{\left|s_{i} \| r_{j}\right|}
$$

where $s_{i}$ represents the learner's space vector, and $r_{j}$ represents the learner's candidate resource. The angle cosine of the two vectors is obtained by the formula. The larger the 
$\operatorname{sim}\left(s_{i}, r_{j}\right)$, the greater the similarity between the two, otherwise the similarity is smaller, with $0 \leq \operatorname{sim}\left(s_{i}, r_{j}\right) \leq 1$.

3) When faced with many resources with the same or similar content, we need to distinguish them by the quality of the resources. The quality of the resources is determined by the amount of playback, favorites, downloads, and user reviews. In order to increase the exposure of new resources, we set a period of time, if within this period, we set the resource quality calculation formula (3):

$$
q u a\left(r_{j}\right)=1
$$

Otherwise, the formula for the quality of resources is shown in formula (4):

$$
q u a\left(r_{j}\right)=\frac{L_{j} * \lambda_{1}}{L}+\frac{S_{j} * \lambda_{2}}{S}+\frac{D_{j} * \lambda_{3}}{D}+\frac{P_{j} * \lambda_{4}}{P}
$$

Among them, $L_{j}, S_{j}, D_{j}, P_{j}$ represent the browse, collection, download, and user comments of the resource $r_{j}$; $L, S, D$, and $P$ represent the maximum browse, collection, download, and user comments of the resource's category; $\lambda_{1}, \lambda_{2}, \lambda_{3}$, and $\lambda_{4}$ are the weights of each evaluation item, and $\lambda_{1}+\lambda_{2}+\lambda_{3}+\lambda_{4}=1$. It can be seen that the matching degree between learner needs and learning resources can be calculated by formula (5):

$$
\operatorname{mat}\left(s_{i}, r_{j}\right)=\operatorname{sim}\left(s_{i}, r_{j}\right) * q u a\left(r_{j}\right)
$$

Finally, the matching degree of each candidate resource is sorted. Recommend resources to target learners according to the degree of fit.

\section{CONCLUSION}

The emergence of the Internet and the era of big data have led to a major change in educational philosophy, from an educator-centric to a learner-centric, the learning mode of learners has also changed from traditional education to personalized education, from difficult to obtain learning resources to difficult to obtain needed learning resources from massive learning resources. The application of personalized recommendation method in the field of education can help learners find the resource information they need in the massive learning resources. Aiming at the cold start problem of current personalized learning resource recommendation methods, this paper starts from the basic personal information of learners at the time of registration to find learners with similar identity background and hobbies for recommendation. To solve the problem of "uneven quality" of recommendation resources, the quality of learning resources is added into the personalized learning model.

In the future, we will further explore key technologies such as big data mining and personalized recommendation algorithms to promote continuous research in this field.

\section{CONFLICT OF INTEREST}

The authors declare no conflict of interest.

\section{AUTHOR CONTRIBUTIONS}

Lijuan Zhou and Feifei Zhang conducted the research and wrote the paper; Shudong Zhang consulted the relevant literature and proposed amendments to the paper; Min xu scrutinized the final revision of the paper; all authors had approved the final version.

\section{ACKNOWLEDGMENT}

In the process of writing a thesis and collecting information, the information of seniors helped me a lot, thanks again. This research was supported by National Key R\&D Program of China(2019YFB1406301).

\section{REFERENCES}

[1] N. Geng, "Research on pushing personalized learning resources based on analysis of student evaluation data," Jiangnan University, 2019.

[2] M. X. Zhang, Li Yi, and Zhou Qin, "Design and implementation of network-based personalized learning system," China Medical Education Technology, 2004, no. 6.

[3] Q. Shi, "Design and implementation of personalized learning resource recommendation system based on user interest preferences," Central China Normal University, 2018.

[4] X. M. Li, "What else can I say about Mu class?" China Education Network, 2015, no. 9, pp. 12-13.

[5] M. Li, "Research and design of learning platform based on personalized resource recommendation," Sichuan: Southwest University of Science and Technology, 2015, Neural Networks for Optimization and Signal Processing, 1st ed, Chichester, U.K.: Wiley, 1993, ch. 2, pp. 45-47.

[6] S. B. Aher and L. M. R. J. Lobo, "Combination of machine learning algorithms for recommendation of courses in e-learning system based on historical data," Knowledge-Based Systems, 2013, vol. 51, pp. 1-14.

[7] K. I. Ghauth and N. A. Abdullah, "Learning materials recommendation using good learners' ratings and content-based filtering," Educational Technology Research and Development, 2010, vol. 58, no. 6, pp. 711-727.

[8] M. K. Khribi, M. Jemni, and O. Nasraoui, "Recommendation systems for personalized technology-enhanced learning," Ubiquitous Learning Environments and Technologies, Springer, Berlin, Heidelberg, 2015, pp. 159-180.

[9] M. Salehi et al., "Personalized recommendation of learning material using sequential pattern mining and attribute based collaborative filtering," Education and Information Technologies, 2014, vol. 19, no. 4, pp. 713-735.

[10] K. G. Saranya and G. S. Sadasivam, "Personalized news article recommendation with novelty using collaborative filtering based rough set theory," Mobile Networks and Applications, 2017, vol. 22, no. 4, pp. 719-729.

[11] S. Bhaskaran and B. Santhi, "An efficient personalized trust based hybrid recommendation (tbhr) strategy for e-learning system in cloud computing," Cluster Computing, 2019, vol. 22, no. 1, pp. 1137-1149.

[12] L. Wang et al., "Cross-domain personalized learning resources recommendation method," Mathematical Problems in Engineering, 2013.

[13] S. Shishehchi et al., "A proposed semantic recommendation system for e-learning: A rule and ontology based e-learning recommendation system," presented at 2010 International Symposium on Information Technology, IEEE, 2010.

[14] G. Q. Pan, "Research on recommendation service of personalized learning resources," Journal of Kashgar University, 2019, vol. 40, no. 03, pp. 116-120.

[15] L. P. Tang, "Research on the recommendation method of learning resources based on ubiquitous learning," Tianjin University of Technology, 2019, An Introduction to Signal Detection and Estimation, New York: Springer-Verlag, 1985, ch. 4.

Copyright (C) 2021 by the authors. This is an open access article distributed under the Creative Commons Attribution License which permits unrestricted 
use, distribution, and reproduction in any medium, provided the original work is properly cited (CC BY 4.0).

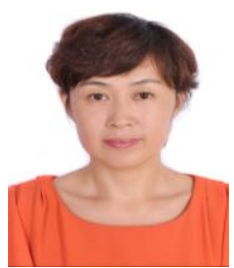

Lijuan Zhou was graduated from Harbin Engineering University with a doctorate degree in computer science and technology in 2004; she was promoted to professor in 2004. In recent years, he has been mainly engaged in research and teaching of data warehouse and business intelligence, data mining and intelligent analysis, database systems and applications.

She is a professor in the School of Information Engineering, Capital Normal University. And hosted the National Science and Technology Personnel Service Enterprise Action Plan Project of the
Ministry of Science and Technology, the National Science and Technology Support Project, the Beijing Municipal Education Commission Science and Technology Development Plan, the Provincial Key Scientific and Technological Research Project, the Natural Science Foundation, Youth Fund, Science and Technology Research Project, and the Municipal Key Scientific and Technological Project More than 20 scientific research topics such as subject reserve leader funds, have achieved important results, won 6 provincial and municipal scientific and technological progress awards, 4 national invention patents, and more than 20 software copyrights. More than 60 related academic papers were published at academic conferences, of which more than 40 were included in three major searches. 